\title{
Relativistic electron loss due to ultralow frequency waves and enhanced outward radial diffusion
}

\author{
T. M. Loto'aniu, ${ }^{1,2,4}$ H. J. Singer, ${ }^{2}$ C. L. Waters, ${ }^{3}$ V. Angelopoulos, ${ }^{4}$ I. R. Mann, ${ }^{5}$ \\ S. R. Elkington, ${ }^{6}$ and J. W. Bonnell ${ }^{7}$ \\ Received 31 May 2010; revised 1 September 2010; accepted 9 September 2010; published 21 December 2010.
}

[1] Using the THEMIS and GOES satellites and ground-based magnetometers, the loss of outer zone radiation belt electrons through the magnetopause in response to ultralow frequency (ULF) waves is examined. A 2 orders of magnitude decrease in $>2 \mathrm{MeV}$ electron flux observed at geosynchronous orbit, starting at 00 UT on 25 June 2008, is attributed to a rapid $(1-4 \mathrm{~h})$ nonadiabatic loss process. ULF waves were observed by the THEMIS-A, -D, and -E probes in the afternoon-to-dusk sector from the magnetopause to geosynchronous altitude. Estimates of the electron resonant energies indicate strong drift resonant interactions occurring between the energetic electrons and the observed waves. The rate of outward radial diffusion was estimated for $\mathrm{MeV}$ electrons using the observed ULF wave azimuthal electric field and compressional magnetic field and the diffusion time $(\sim 2.5 \mathrm{~h})$ was found to be in good agreement with the observed time for nonadiabatic flux decreases at geosynchronous orbit. The magnetopause was compressed inside of its nominal position because of increased solar wind dynamic pressure. The electron loss is interpreted as a combination of magnetopause shadowing (from the compressed magnetosphere) and enhanced outward diffusion from ULF wave-particle drift resonant interactions. The enhanced day-night asymmetry of the $\mathrm{MeV}$ electron drift path from the compression suggests that enhanced losses may have also occurred around local noon as well as in the afternoon-to-dusk sector.

Citation: Loto'aniu, T. M., H. J. Singer, C. L. Waters, V. Angelopoulos, I. R. Mann, S. R. Elkington, and J. W. Bonnell (2010), Relativistic electron loss due to ultralow frequency waves and enhanced outward radial diffusion, J. Geophys. Res., 115, A12245, doi:10.1029/2010JA015755.

\section{Introduction}

[2] The mechanisms responsible for producing the dynamics observed in Earth's outer radiation belt electron fluxes are not completely understood. Although many studies have investigated electron acceleration, more recent studies have emphasized the importance of loss processes in determining the overall flux levels [e.g., Reeves et al., 2003; Meredith et al., 2003; Summers and Thorne, 2003; Loto'aniu et al., 2006b; Summers et al., 2007a, 2007b;

\footnotetext{
${ }^{1}$ Cooperative Institute for Research in Environmental Sciences, University of Colorado at Boulder, Boulder, Colorado, USA.

${ }^{2}$ Space Weather Prediction Center, National Oceanic and Atmospheric Administration, Boulder, Colorado, USA.

${ }^{3}$ Center for Space Physics, School of Mathematical and Physical Sciences, University of Newcastle, Newcastle, New South Wales, Australia.

${ }^{4}$ Institute for Geophysics and Planetary Physics, University of California, Los Angeles, California, USA.

${ }^{5}$ Department of Physics, University of Alberta, Edmonton, Alberta, Canada.

${ }^{6}$ Laboratory for Atmospheric and Space Physics, University of Colorado at Boulder, Boulder, Colorado, USA.

${ }^{7}$ Space Sciences Laboratory, University of California, Berkeley, California, USA.

Copyright 2010 by the American Geophysical Union. 0148-0227/10/2010JA015755
}

Ohtani et al., 2009; Xiao et al., 2009a]. During the main phase of a geomagnetic storm the ring current build ups, which decreases the background magnetic field strength in the inner magnetosphere. This process leads to outward radial motion of relativistic electron drift paths in order to conserve the third adiabatic invariant, and produces an adiabatic decrease in flux levels often referred to as the Dst effect [e.g., Kim and Chan, 1997; Millan and Thorne, 2007]. In the outer radiation belt, flux decreases resulting in net electron losses are usually attributed to electromagnetic ion cyclotron (EMIC) and very low frequency (VLF) waveparticle interactions that result in pitch angle diffusion into the atmosphere [e.g., Summers and Thorne, 2003; Loto'aniu et al., 2006b; Summers et al., 2007a; Xiao et al., 2009b], and/or magnetopause shadowing due to particle motion on open drift shells [e.g., Ohtani et al., 2009].

[3] When the magnetopause is compressed, losses in the outer radiation belt are thought to be enhanced [e.g., Kim et al., 2008]. Particles previously on closed drift paths now find themselves on open drift paths due to the compression [Green et al., 2004]. The compression related losses can create electron phase space density that decreases with increasing radial distance, which produces outward radial motion from the outer radiation belt leading to further losses [Shprits et al., 2006]. However, Green et al. [2004] 
found that intense losses during the main phase of a storm cannot be produced by magnetopause encounters alone, since losses extend much farther into the heart of the radiation belt than the estimated storm time magnetopause location.

[4] As for wave-particle interactions causing losses, EMIC wave-electron minimum resonant energies typically fall below $2 \mathrm{MeV}$ only when the ratio of the plasma frequency to equatorial cyclotron frequency is above 10 [Meredith et al., 2003]. Such conditions are found in regions of high plasma density and low magnetic field strength such as the duskside plasmapause or within plasma plumes at high $L$ [Meredith et al., 2003]. EMIC wave-particle scattering generally cannot explain radiation belt depletions at hundreds of keV [e.g., Meredith et al., 2003; Summers and Thorne, 2003; Loto'aniu et al., 2006b]. Studies of losses due to VLF wave scattering suggest that losses can occur throughout the outer radiation belt [e.g., Albert, 2005; Summers et al., 2007a]. However, VLF chorus scattering of $\geq 1 \mathrm{MeV}$ electrons in the outer radiation belt is generally slow compared to EMIC wave scattering [e.g., Summers et al., 2007a] and cannot explain rapid electron losses. More recently, Summers et al. [2008] studied whistler mode extremely low frequency (ELF) hiss in plasmaspheric drainage plumes and found them to be effective in inducing relativistic electron loss, given sufficiently strong wave power.

[5] Rapid outer radiation belt electron losses have been observed simultaneously with adiabatic changes in the magnetosphere [e.g., Selesnick, 2006; Millan et al., 2007; Shprits et al., 2006; Ukhorskiy et al., 2006]. Selesnick [2006] studied storm time relativistic loss events and found that loss rates typically increase during the main phase of a storm and that depletion of the prestorm radiation belt can occur on a time scale $\sim 1 \mathrm{~h}$. Millan et al. [2007] used balloon observations of X-ray emissions and simultaneous observations of $>2 \mathrm{MeV}$ electron fluxes at geosynchronous orbit to study a substorm related relativistic electron precipitation event. They concluded that the electrons were lost to the atmosphere early in the flux depletion event, during a period of magnetic field stretching in the tail. Ukhorskiy et al. [2006] investigated the evolution of the outer radiation belt during a geomagnetic storm event using test particle simulation. They found that the diamagnetic influence of the partial ring current (Dst effect) leads to expansion of electron drift orbits such that their paths intersect the magnetopause leading to rapid electron losses $(\sim 2.5 \mathrm{~h}$ after storm onset).

[6] Wave-particle interactions in the radiation belt are often studied as a diffusion process. Ultralow frequency (ULF) waves interacting with radiation belt particles can cause inward and/or outward radial diffusion. The direction of particle diffusion depends on the slope of relativistic electron phase space density. In the magnetosphere a positive slope (outward radial gradient) will result in inward diffusion toward Earth, and a negative slope (inward radial gradient) will result in outward radial diffusion toward the magnetopause. Recent studies suggest that for both quiet and storm times, the electron phase space density around geosynchronous orbit and beyond is negative for $\mathrm{MeV}$ electrons [Turner and Li, 2008; Chen et al., 2005, 2006, 2007; Iles et al., 2006]. However, Iles et al. [2006] found that it is possible to have inward radial diffusion at low energies and outward radial diffusion at higher energies.

[7] Green and Kivelson [2004] used polar observations to study radiation belt electron phase space density profiles. They suggest that in the presence of strong ULF wave power, local phase space density peaks would cause outward radial diffusion, increasing electron flux at geosynchronous and broadening the phase space density peak to larger $L$ values. Varotsou et al. [2005] presented the first simulation results for electrons in the outer radiation belt near geosynchronous, where radial diffusion and resonant interactions with the whistler mode chorus outside the plasmasphere are taken into account. Their simulations using the Salammbø code showed that at high energies radial diffusion may transport particles outward and contribute to electron deceleration. This was further confirmed by a three-dimensional simulation of outer radiation belt electron dynamics including cross-diffusion terms [Xiao et al., 2010]. Varotsou et al. [2005] suggested that wave acceleration in the outer region must overcome losses caused by outward radial diffusion.

[8] Simulation studies of radial diffusion during storms have included varying the outer boundary conditions using satellite observations [e.g., Brautigam and Albert, 2000; Miyoshi et al., 2003; Shprits et al., 2006]. Results from Brautigam and Albert [2000] and Miyoshi et al. [2003] indicate that outward radial diffusion may significantly contribute to the variability of the radiation belt. More recently, Shprits et al. [2006] used HEO, SAMPEX, and CRRES observations to suggest that storm main phase radiation belt electron depletions occur when the magnetopause is compressed and the $\mathrm{Kp}$ index (used as a proxy for ULF wave activity) is high. They used simulations to show that negative gradient in phase space density created by losses to the magnetopause and subsequent outward radial diffusion, can contribute to main phase depletions of the radiation belts.

[9] In this study, observations from the THEMIS [Sibeck and Angelopoulos, 2008] and GOES satellites and CARISMA ground magnetometer stations [e.g., Mann et al., 2009] are used to study the relationship between ULF waves and the loss of radiation belt outer zone relativistic electrons through the magnetopause on 25 June 2008. A scenario is presented to explain the observations whereby a combination of magnetospheric compression and ULF waves near the magnetopause enhances losses through the magnetopause. The mechanism is presented in section 2 using schematics to show how relativistic electron phase space density might evolve under the aforementioned conditions. In section 3.1 the solar wind conditions and GOES observations are presented, while the location of the magnetopause is discussed in section 3.2. The orbits of relevant spacecraft around 25 June 2008 are briefly explained in section 3.3 .

[10] The THEMIS relativistic electron flux observations are shown in section 3.4. Currently, studies are being pursued to determine the absolute detector flux levels measured by the Solid State Telescope (SST) instruments [Angelopoulos, 2008] on the THEMIS probes and crosscalibration with other spacecraft. The results of that work will not affect the results of this study, which rely on relative changes of observed fluxes as a function of time during the 


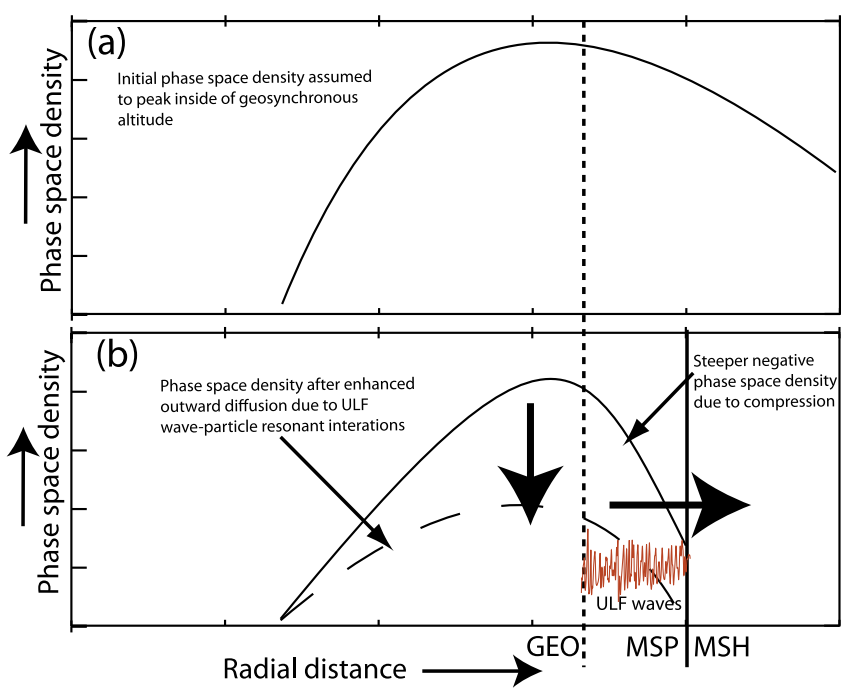

Figure 1. Scenario for enhanced loss through the magnetopause. GEO and vertical dotted line refer to geosynchronous altitude, MSP is the magnetosphere and MSH the magnetosheath. (a) The initial phase space density. (b) Enhanced solar wind pressure compresses the magnetosphere causing enhanced magnetopause shadowing and steeper negative phase space density at higher altitudes (solid curve). Consequently, electrons diffuse toward the magnetopause while encountering ULF waves (in red) and undergo wave-particle drift-resonance which enhances outward diffusion leading to rapid losses through the magnetopause and subsequent decrease in the outer radiation belt phase space density (dashed curve).

event. Calculations of the actual phase space density are beyond the scope of this study.

[11] The observed time scale for relativistic losses is shown in section 3.5. In section 3.6, THEMIS in situ and CARISMA ground-based observations of ULF waves are presented. Sections 4 and 5 are devoted to calculating wave spectral properties, resonant energies and diffusion time scales assuming wave-particle drift resonant interactions. The results are discussed and summarized in sections 6 and 7 .

\section{Scenario for Enhanced Loss Through Magnetopause}

[12] The mechanism of relativistic flux loss due to a combination of a magnetospheric compression and ULF wave-particle interactions resulting in enhanced outward diffusion [Shprits et al., 2006] requires the outer zone phase space density to evolve according to specific criteria. The observations shown later in the paper are consistent with the following hypothetical scenario. Figure la shows the initial phase space density, which is assumed to peak for 1-2 MeV electrons near geosynchronous-altitude (GEO). In Figure 1b, the pressure pulse impacting on the magnetosphere results in compression that moves the magnetopause closer to Earth and some electrons previously on closed drift paths can be lost from the magnetosphere due to magnetopause shadowing. During magnetospheric compression, the day-night magnetic asymmetry is enhanced and as a consequence the area of closed drift orbits shrinks [Ohtani et al., 2009]. This results in a steeper negative phase space density gradient near and beyond geosynchronous (decreasing phase space density with increasing radial distance) for constant first adiabatic invariant, which forces relativistic electrons to diffuse outward toward the magnetopause. In order to conserve the first adiabatic invariant, particles on the negative slope of phase space density must lose energy while diffusing outward. As the electrons diffuse toward the magnetopause they encounter ULF waves and may undergo wave-particle drift-resonance, which further enhances outward diffusion causing rapid relativistic electron loss through the magnetopause.

\section{Results for 25 June 2008 Event}

\subsection{Solar Wind and GOES Observations}

[13] The general solar wind and geomagnetic conditions both at geostationary orbit and on the ground from 24-28 June 2008 are presented in Figure 2. Shown top to bottom are Sym-H (1 min resolution Dst), Kp*10, solar wind $\mathrm{Bz}$ (GSM), solar wind pressure (Pdyn), solar wind velocity (Vsw), total magnetic field at geosynchronous (Ht), $>2 \mathrm{MeV}$ electron fluxes measured by GOES-12, and the magnetopause stand off distance calculated using the Shue et al. [1998] model and $1 \mathrm{~h}$ averaged Pdyn and Bz. The solar wind parameters and geomagnetic indices are taken from the NASA CDAWeb Web site (http://cdaweb.gsfc.nasa.gov/), while GOES data come from the Space Weather Prediction Center online data server (http://www.swpc.noaa.gov/Data/ index.html). Close to 00 UT on 25 June 2008 the GOES satellites (GOES-10, -11 and -12) observed a 2 orders of magnitude drop in relativistic electron fluxes over about $1-4 \mathrm{~h}$ (see section 3.5). The flux levels at geosynchronous do not return to their previous levels for two and one-half days.

[14] An enhanced pressure region can be seen in the Pdyn panel beginning 20 UT on 24 June and lasting for the duration of the interval shown in Figure 2. The GOES-12 Ht panel shows large variations in field values at geosynchronous during the event. The first magnetic field amplitude spike coincides with the beginning of the enhanced pressure and the storm sudden commencement (SSC) seen in the Sym-H panel. However, the storm that follows is moderate with Sym-H remaining above $-40 \mathrm{nT}$ and the $\mathrm{Kp}$ index remains relatively low.

[15] The magnetic field amplitude observed by GOES undergoes periodic changes due to the day-night magnetic asymmetry. Originally, relativistic electrons undergoing adiabatic motion are observed by GOES with a similar periodic change in flux levels. However, in Figure 2 on 25 June the electron fluxes remain low while Ht shows periodic amplitude changes that move above and below 95-120 nT, the nominal field range observed before the event occurred. These observations suggest that electrons were lost at geosynchronous orbit during the two and one-half days interval from about $00 \mathrm{UT}$ on 25 June to $\sim 12$ UT on 27 June.

\subsection{Magnetopause Shadowing}

[16] The magnetopause stand off distance shown at the bottom of Figure 2 suggest the magnetopause compressed to $\sim 8.8 R_{E}$ at $\sim 23: 30$ UT on 24 June and $\sim 8.5 R_{E}$ at $\sim 03: 30 \mathrm{UT}$ on 25 June. Using higher-resolution (1 $\mathrm{min})$ solar wind data, the smallest standoff distances (not shown) near midnight 


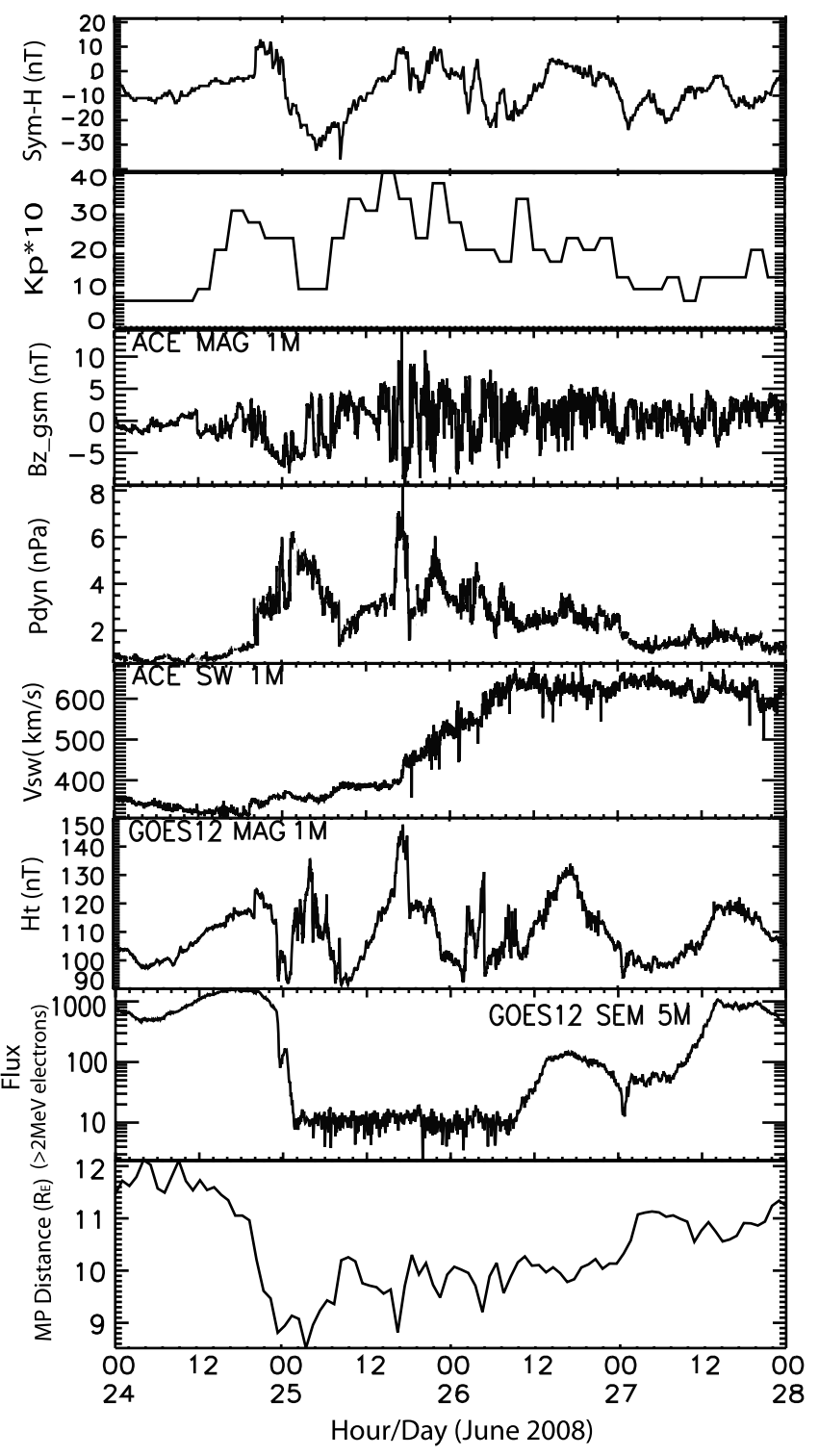

Figure 2. Geomagnetic indices, solar wind, and GOES data (see text for description) on 24-28 June 2008. (bottom) The magnetopause stand off distance calculated using the Shue et al. [1998] magnetopause model and $1 \mathrm{~h}$ averaged Pdyn and solar wind $\mathrm{Bz}$ as inputs.

UT were $\sim 8.3 R_{E}$ at $\sim 21: 40$ UT on 24 June and $\sim 8.3 R_{E}$ at $\sim 00$ UT on 25 June. The magnetospheric compressions cause enhanced magnetopause shadowing. As stated in section 2, magnetospheric compression enhances the daynight magnetic asymmetry, shrinking the area of closed drift orbits [Ohtani et al., 2009]. This creates a negative phase space density gradient near geosynchronous altitudes and beyond. Hence, around midnight on 24 June, particles diffuse outward toward the magnetopause due to the negative phase space density created by the loss of particles from magnetopause shadowing.

\subsection{Spacecraft Locations}

[17] Figure 3 shows THEMIS and GOES orbit trajectories for 24-25 June in the X-Y GSE coordinate plane. Each spacecraft trajectory is color coded with symbols indicating the position of the spacecraft at 00 UT on 25 June. Figure 3 was generated using the NASA SSCWeb 3-D Orbit Viewer (http://sscweb.gsfc.nasa.gov/tipsod/), assuming a nominal Pdyn $\sim 2 \mathrm{nPa}$ to estimate the magnetopause and bow shock locations using the Roelof and Sibeck [1993] and Fairfield [1971] models, respectively. At 00 UT on 25 June, THM-A (THEMIS-A), $-\mathrm{D}$ and $-\mathrm{E}$ were located in the afternoon sector ( 16-17 MLT) near apogee in the outer magnetosphere, while THM-B and $-\mathrm{C}$ are both in the solar wind. The THM-A, -D and -E spacecraft were well located for observational comparisons with the GOES spacecraft.

\subsection{THEMIS Particle Observations}

[18] Figure 4 shows daily averaged omnidirectional $\sim 0.65 \mathrm{MeV}$ electron fluxes observed by the THM-E Solid State Telescope (SST) instrument [Angelopoulos, 2008] as a function of $L$ value during 18-30 June. The fluxes were mapped to the equator using a simple assumption of a flat pitch angle distribution with values scaled by $B_{m} / B_{e q}$, where $B_{m}$ is the total magnetic field at THM-E and $B_{e q}$ is the equatorial field assuming a dipole configuration. The $0.65 \mathrm{MeV}$ channel responds not only to primary and secondary electrons of a broad energy above $\sim 0.35 \mathrm{MeV}$, but also to ions $>0.7 \mathrm{MeV}$ that penetrate the electron foil in front of the electron detector. In the outer radiation belt ( $L \geq \sim 3.5$ during solar minimum) electron fluxes dominate and proton contamination is small, hence, the temporal variation of the flux observed is an accurate representation of outer zone electron variations. At $L \sim 3$ the $\mathrm{MeV}$ proton fluxes are significant and electron fluxes are low, resulting in the observed second flux contamination peak at that location.

[19] Still, the outer zone radiation belt is clearly visible in Figure 4. Note, outer zone fluxes peak at $L$ values slightly higher than that previously observed by CRRES and

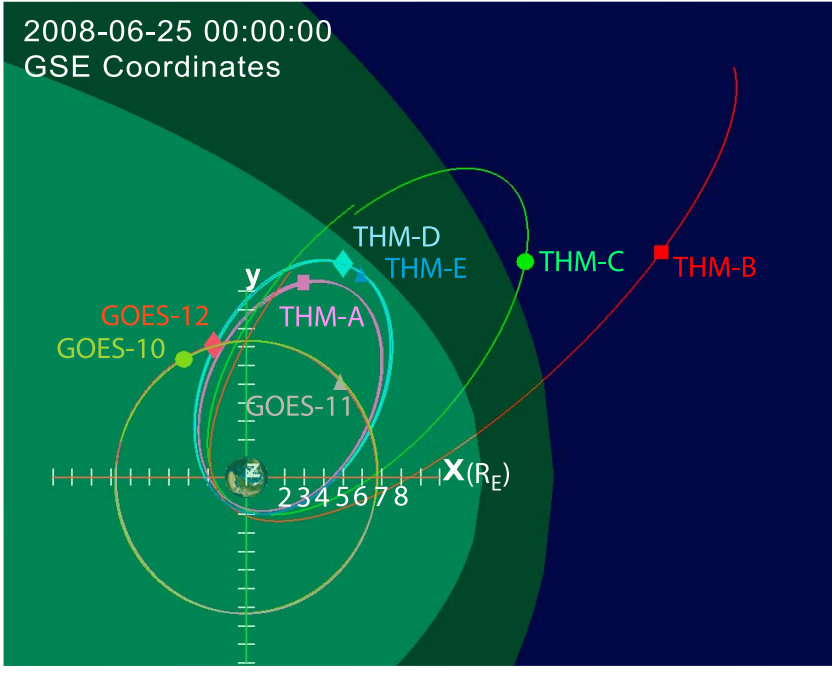

Figure 3. THEMIS and GOES orbits in GSE X-Y plane for 24-25 June 2008. The figure was generated using the NASA SSCWEB 4D Orbit Viewer (http://sscweb.gsfc. nasa.gov/tipsod/), and the dark blue region to the right represents outside of the magnetosphere, while the dark green and light green regions correspond to the magnetosheath and magnetosphere, respectively. 


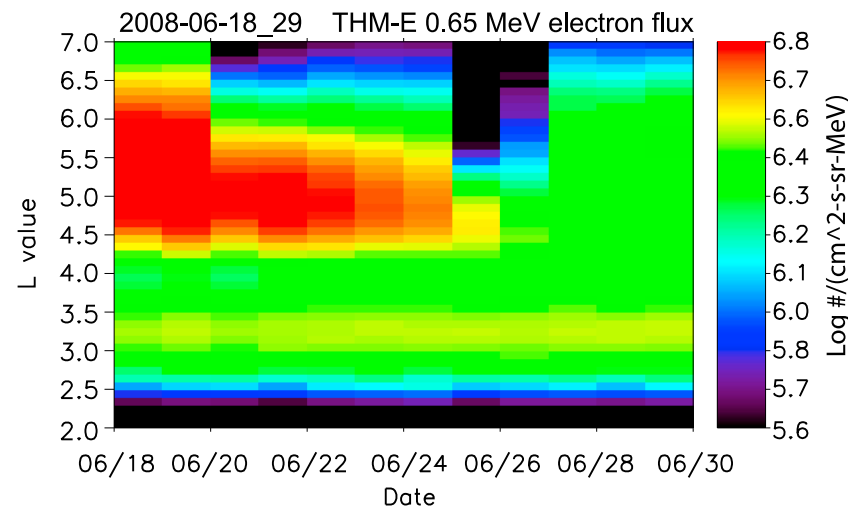

Figure 4. THM-E SST instrument $\sim 0.65 \mathrm{MeV}$ electron daily averaged fluxes during 18-30 June 2008.

SAMPEX [ $L i$ et al., 2001; Li and Temerin, 2001]. This is most likely due to a combination of assuming a dipole field model when mapping to the equator, and the fact that these observations were taken during solar minimum when the magnetosphere is usually inflated. It is clear from Figure 4 that flux levels in the outer zone (for example, $L \sim 4.5-5.5$ ) decreased starting on 25 June and remained low even after 28 June when GOES (see Figure 2) observed fluxes returning to prestorm levels. The combined THEMIS and GOES spacecraft observations provide strong evidence of electron loss in the outer zone with most losses occurring on 25 June.

\subsection{Time Scale for Relativistic Losses}

[20] Accurately estimating the time scale for nonadiabatic losses using observations alone is not possible. However, some understanding of the time scale can be gained by comparing the variations in local magnetic field strength to that of the relativistic electron fluxes. Figure 5 shows, from top to bottom, the solar wind Pdyn, Sym-H and GOES-12 total magnetic field (Ht) and relativistic electron flux over a $42 \mathrm{~h}$ period on 24-25 June. The horizontal red line is drawn at the $110 \mathrm{nT}$ average background magnetic field value in order to compare characteristics of the event before and during the pressure enhancement period. The green line indicates when the background field first returns to $\sim 110 \mathrm{nT}$ amplitude.

[21] Shortly before midnight UT on 24 June, GOES-12 measured a sudden decrease in relativistic fluxes followed immediately by a small increase at midnight (blue line). The same variation was observed in $\mathrm{Ht}$ indicating that the flux changes were probably due to adiabatic changes. Shortly after 00 UT, relativistic fluxes decrease again but by $\sim 01: 30$ UT (green line), the field is increasing but flux levels remain low. The time interval between the vertical blue and green lines in Figure 5 suggest enhanced nonadiabatic losses over $\sim 90 \mathrm{~min}$.

[22] This time scale was also suggested by the GOES-10 observations (not shown). However, similar analysis of the GOES-11 observations (not shown) imply a slower loss time of possibly 3-4 h. The GOES-10 and GOES-12 satellites are separated by only $\sim 1$ h of MLT, while GOES-11 trails GOES-12 by about $4 \mathrm{~h}$ MLT. Hence, differences in observed loss time may be due to local time affects. The combined GOES observations suggest that geosynchronous
$\mathrm{MeV}$ electrons were nonadiabatically depleted, starting at 00 UT on 25 June 2008 , over a period of $1-4 \mathrm{~h}$.

\subsection{ULF Wave Observations}

\subsubsection{THEMIS Spacecraft Observations}

[23] The THM-A fluxgate magnetometer [Auster et al., 2008] measured ULF waves, shown in Figure 6a on 25 June. THM-D and THM-E entered the magnetosphere between 02 and 03 UT and immediately observed ULF waves (Figures $6 \mathrm{~b}$ and $6 \mathrm{c}$ ). The ULF waves were also observed (Figures 6d-6f) by the THM-A, -D and -E electric field instruments (EFIs) [Bonnell et al., 2008]. For the electric fields, the spacecraft axial electric field component was replaced with values determined from the 2-D spacecraft spin plane electric field components using $\vec{E} \cdot \vec{B}=0$, which assumes electromagnetic plane wave propagation [Bonnell et al., 2008]. The THEMIS probes were located in the afternoon to dusk sectors and observed the ULF wave along their trajectories to lower altitudes.

[24] Figures 6g-6j show THM-A field-aligned coordinate (FAC) band limited (1-10 mHz) wave data over 00-04 UT on 25 June. In this FAC system, the $\mathrm{Z}$ component is defined parallel to the background magnetic field vector, $X$ is positive outward from Earth and $\mathrm{Y}$ is positive eastward completing a right-handed system. The magnetic wave amplitude was dominant in the Bx_FAC and Bz_FAC components for all three spacecraft reaching amplitudes over $20 \mathrm{nT}$ peak to peak. Considering the THM-A, -D and -E locations, these components correspond to meridional oscillations and thus to a radial and compressional ULF wave mode magnetic perturbation. For the electric wave amplitudes, the Ex_FAC and Ey_FAC components were dominant and peak-to-peak amplitudes sometimes exceeded $4 \mathrm{mV} / \mathrm{m}$.

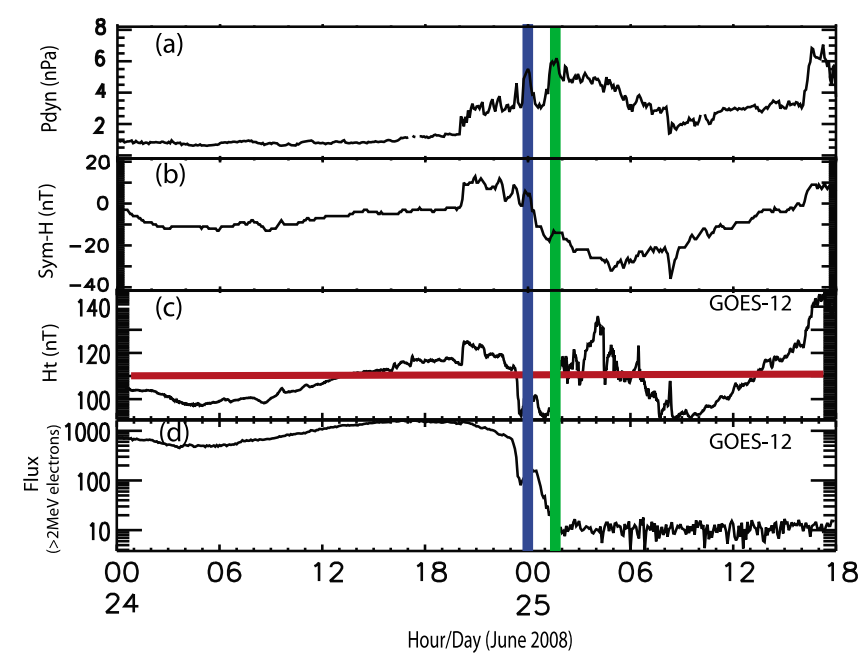

Figure 5. An expanded view of solar wind dynamic pressure (Pdyn), Sym-H, and GOES total magnetic field (Ht) and $\mathrm{MeV}$ electron data during 24-25 June 2008. The horizontal red line is drawn at the $110 \mathrm{nT}$ background field value in order to compare characteristics of the event before and during the pressure enhancement period. The blue line indicates a small adiabatic increase in the $\mathrm{MeV}$ fluxes, and the green line indicates when the background field first returns to $\sim 110 \mathrm{nT}$ amplitude. 

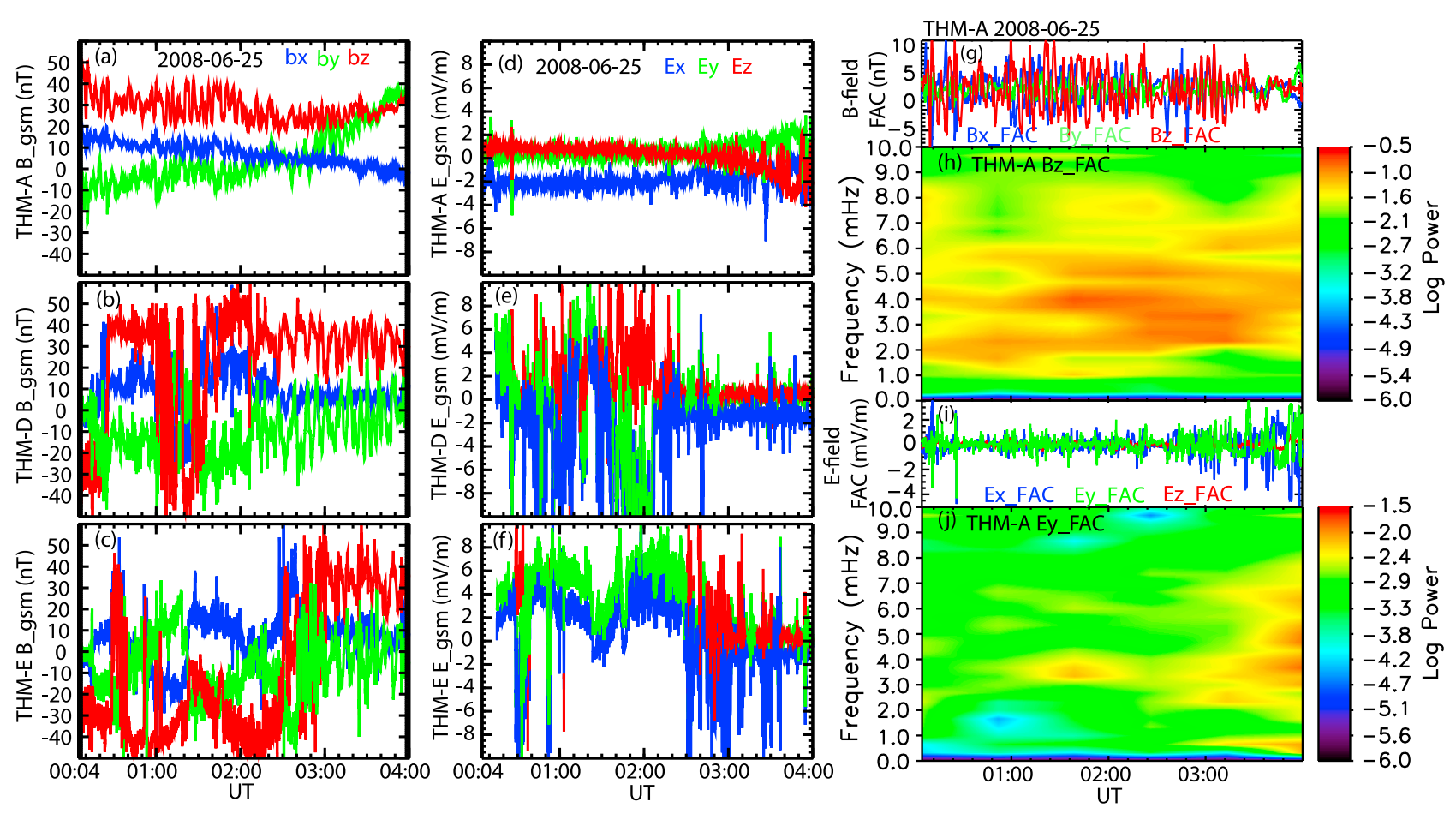

Figure 6. (a-f) The magnetic and electric field $3 \mathrm{~s}$ resolution time series data from THM-A, $-\mathrm{D}$, and $-\mathrm{E}$ during $00-04 U T$ on 25 June 2008. $(\mathrm{g}-\mathrm{j})$ The band limited $(1-10 \mathrm{mHz})$ field-aligned coordinate (FAC) THM-A wavefield data, with (g) the wave magnetic field, (h) the corresponding power spectrogram for the magnetic compressional (Bz_FAC) component, (i) wave electric field, and (j) the corresponding power spectrogram for the electric azimuthal (Ey_FAC) component.

[25] The magnetic compressional (Bz_FAC) and electric azimuthal (Ey_FAC) wave power were observed over a broad range of frequencies $(\sim 2-8 \mathrm{mHz})$ in both the magnetic and electric field observations, although, peaks in power were mostly limited to $2-5 \mathrm{mHz}$. When THM-D entered the magnetosphere at $\sim 02$ UT, magnetic wave power (not shown) shifted to slightly lower frequencies in the $1-5 \mathrm{mHz}$ range. These observations of ULF waves are consistent with the model for electron loss presented in section 2 (see Figure 1b). As the electrons diffuse outward they encounter ULF waves all the way to the magnetopause. However, a drift resonance condition must be satisfied for wave-particle interactions. Determining the resonance condition is the focus of section 4 .

\subsubsection{Ground-Based Observations}

[26] The in situ THEMIS spacecraft measurements are somewhat limited in their ability to observe the global properties of ULF waves. Ground-based observations using many magnetometers provide a more global picture of the wave properties. The Canadian Array for Realtime InvestigationS of Magnetic Activity (CARISMA) magnetometer chain [e.g., Mann et al., 2009], shown in Figure 7 was used to obtain the radial distribution of ULF wave power and to gain insight into the wave $m$ number [Olson and Rostoker, 1978]. Estimating wave radial distribution is important to understand if wave power was observed on the negative or positive phase space density slope, and the $m$ number is important for estimating the wave-particle drift resonance condition.

[27] Figures 8a and 8b show ULF wave time series and corresponding power spectra observed at station RANK, respectively. The ground-based D component (blue line in Figure $8 \mathrm{~b}$ ), which corresponds to the poloidal mode in space, has power peaking at about $4 \mathrm{mHz}$ with a value of $\sim 7 \mathrm{nT}^{2} / \mathrm{Hz}$. The RANK station observations of wave frequency are in agreement with the THEMIS observations (Figure 6), being band-limited over about $2-5 \mathrm{mHz}$. This result indicates that the ground station and THEMIS spacecraft observed the same waves, hence, implying low $m$ number ULF waves as high $m$ waves are screened by the ionosphere and not usually observed on the ground [Hughes, 1974].

[28] Figure 8c shows the ground-based ULF wave power with $L$ value for the first $2 \mathrm{~h}$ on 25 June. The wave power was calculated by summing the power spectra between

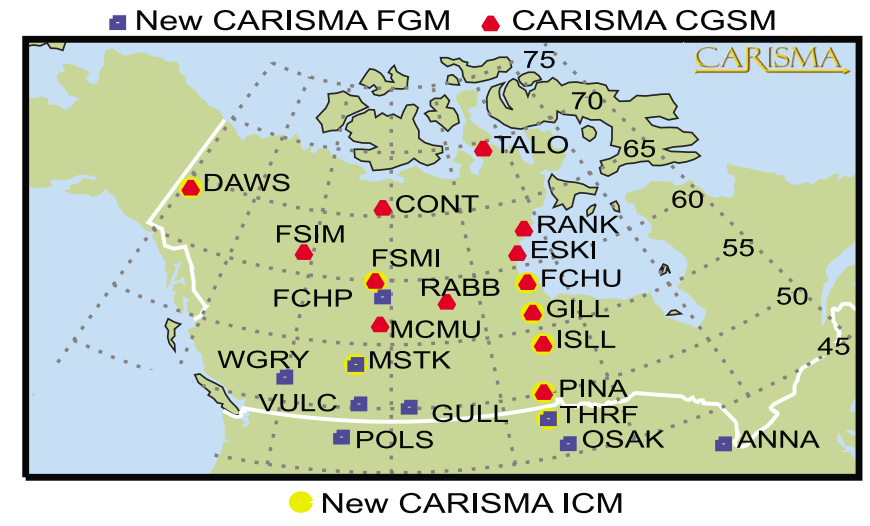

Figure 7. The CARISMA ground-based magnetometer chain. 

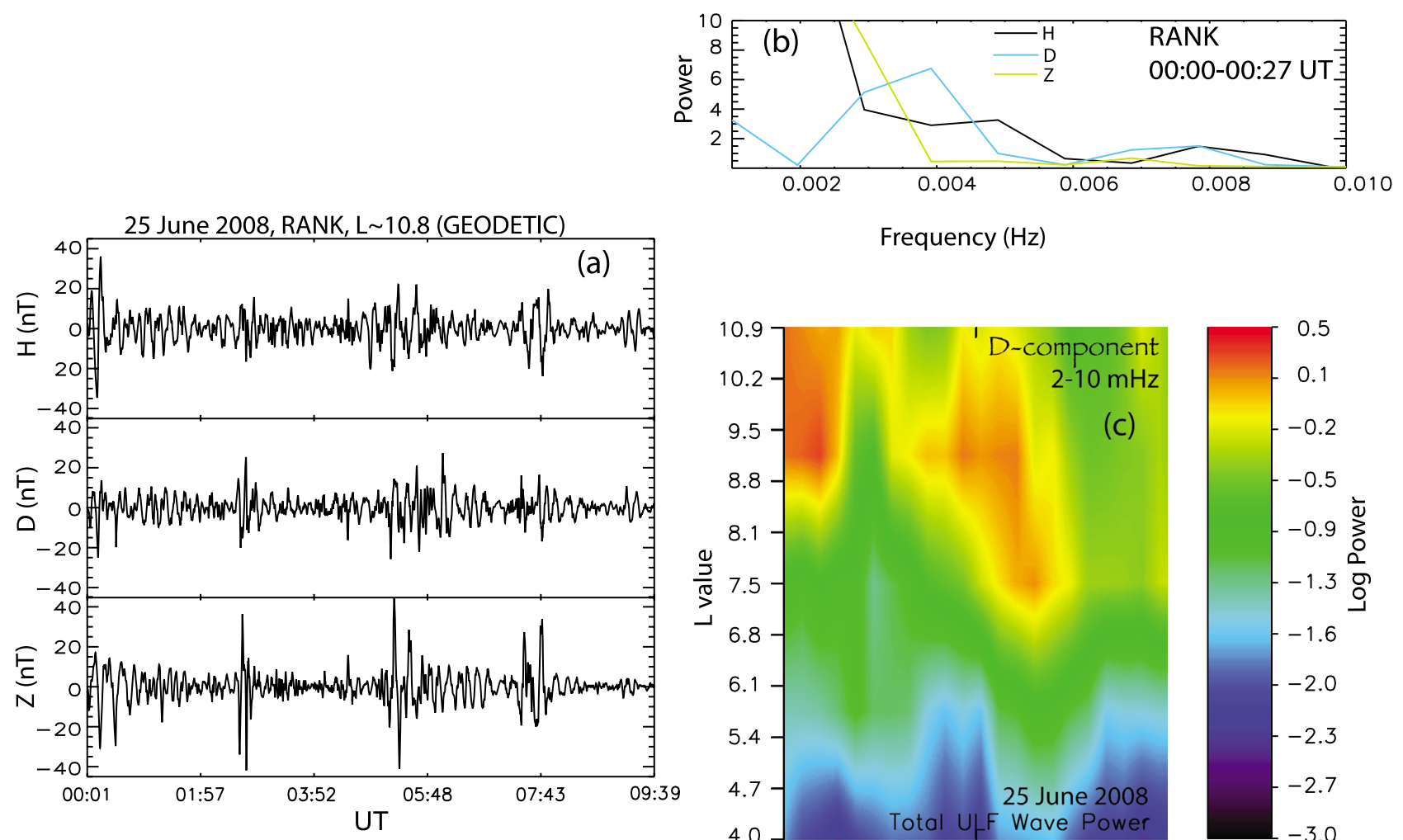

Frequency $(\mathrm{Hz})$

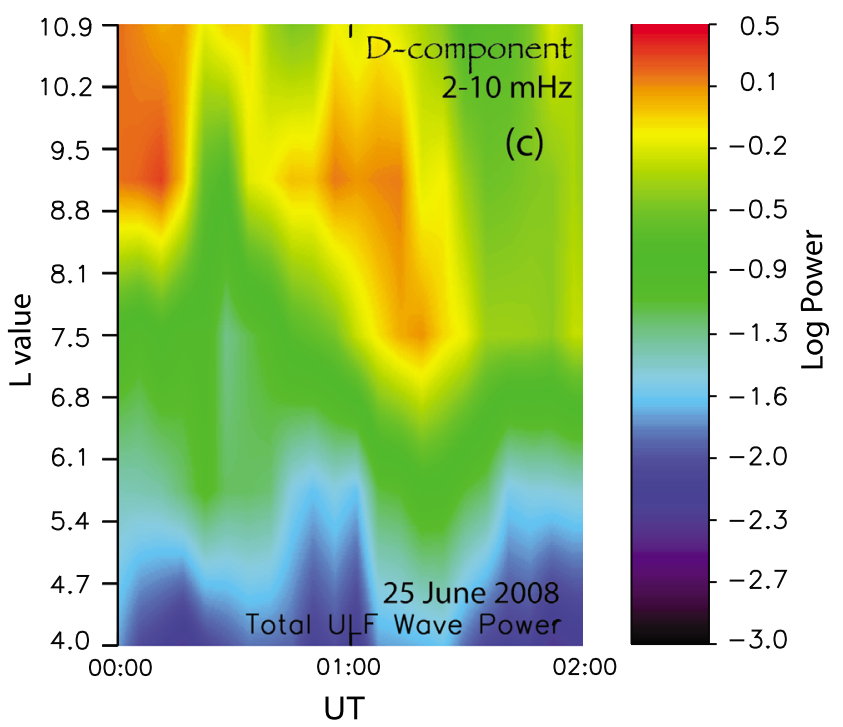

Figure 8. (a) An example of ground-based magnetometer time series observed at station RANK during 25 June 2008 showing ULF waves. (b) Corresponding magnetic power spectrum over $27 \mathrm{~min}$. (c) The ground-based ULF wave power with $L$ value for the first $2 \mathrm{~h}$ on 25 June. The wave power with $L$ value was calculated by summing the power spectra between 2 and $10 \mathrm{mHz}$ of latitudinally spaced ground magnetometer measurements along the Churchill line of the CARISMA array.

2 and $10 \mathrm{mHz}$ of latitudinally spaced ground magnetometer measurements along the Churchill line of the CARISMA array and interpolating between stations using cubic spline interpolation. The spectral results confirm that wave power was present above geosynchronous altitudes and most likely on the negative side of the phase space density gradient following the pressure pulse arrival. This result, given the $L$ range, is consistent with the scenario described in section 2 for electron loss via magnetopause shadowing.

\section{Resonant Energies}

[29] For relativistic electrons, the first adiabatic invariant, $\mu$, can be related to electron kinetic energy, $E_{k}$, by

$$
\mu=\frac{p_{\perp}^{2}}{2 m_{0} B_{0}}, \quad p_{\perp}=p \sin \alpha, \quad p^{2}=\left(E_{k}^{2}+E_{k} 2 m_{0} c^{2}\right) / c^{2},
$$

where $\alpha$ is the electron pitch angle, $m_{0}$ is electron rest mass and $p_{\perp}$ and $p$ are relativistic perpendicular and total momentum, respectively. Assuming $90^{\circ}$ pitch angle electrons and using a nominal magnetic field value of $\sim 110 \mathrm{nT}$, which was the average field value observed by GOES-12 before the flux dropout (see section 3.5), two $\mu$ values $1800 \mathrm{MeV} / \mathrm{G}$ and $5400 \mathrm{MeV} / \mathrm{G}$ were calculated correspond- ing to $1 \mathrm{MeV}$ and $2 \mathrm{MeV}$ electrons at geosynchronous orbit. Assuming $\mu$ remains constant, Figure 9 shows electron energy curves with $L$ value using equation (1) and assuming a dipole field. The energies of 1-2 $\mathrm{MeV}$ electrons at GOES altitudes, if $\mu$ is conserved and assuming outward diffusion due to magnetopause shadowing, would adiabatically decrease to $\sim 400-900 \mathrm{keV}$ by $L \sim 12$.

[30] The wave-particle drift resonance condition for symmetric interactions [e.g., Elkington, 2006] is

$$
\omega=m \omega_{d}
$$

where $\omega_{d}$ is the particle azimuthal drift frequency, $m$ is the azimuthal wave mode number and $\omega$ its frequency. The drift energies, $W_{\text {res }}$, at which radiation belt electrons $(>100 \mathrm{keV})$ will satisfy the resonance condition, assuming a dipole field can be written as

$$
W_{\text {res }}=\frac{\omega}{m} \frac{q B_{s} R_{E}^{2}}{6 P(\alpha) L}+\left[\left(\frac{\omega}{m} \frac{q B_{s} R_{E}^{2}}{6 P(\alpha) L}\right)^{2}+m_{0}^{2} c^{4}\right]^{1 / 2}-m_{0} c^{2},
$$

where $B_{s}$ is the magnetic field at Earth's surface; $R_{E}$ is Earth's radius and $c$ is the speed of light [Ozeke and Mann, 2008]. $P(\alpha)$ is a function of pitch angle and can be approximated by $P(\alpha) \mathrm{C} 0.35+0.15 \sin \alpha$ [Hamlin et al., 1961; Ozeke and Mann, 2008]. 


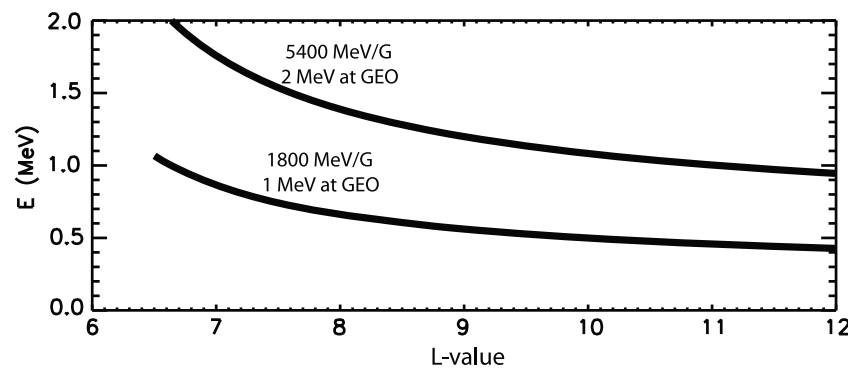

Figure 9. Energy curves with $L$ value for electrons assuming 1 and $2 \mathrm{MeV}$ at geosynchronous altitudes or assuming two different constant first adiabatic invariants $\mu$.

[31] Using equation (3), $90^{\circ}$ pitch angle electrons and the observed ULF wave spectral properties, $W_{\text {res }}$ was estimated at the THM-D and THM-A locations during 00-05 UT on 25 June. The results, shown in Figure 10, assumed that wave $m$ numbers were $m=1$ and $m=2$, which is justified by results in section 3.6.2 where observations by ground magnetometer stations indicate low $m$ numbers. Wave frequencies were obtained from Figure 6. From THM-A observations, wave frequency was taken as $3.5 \pm 0.5 \mathrm{mHz}$. However, at THM-D wave spectral power was observed over a wide frequency range and instead two wave bands were used, at $3.5 \pm 0.5 \mathrm{mHz}$ and $1.5 \pm 0.5 \mathrm{mHz}$. The thickness of the curves in Figure 10 corresponds to $\pm 0.5 \mathrm{mHz}$ spectral width of the waves. These results indicate that drift resonance was satisfied on 25 June for the observed ULF waves consistent with the flux dropout shown in Figure 4.

\section{Radial Diffusion}

[32] In this section, estimates of the rate of radial diffusion using THM-A field observations are presented. For these calculations, we assume that radial diffusion results from symmetric resonant interactions and use equation (2). Under this assumption, the radial diffusion coefficient, $D_{L L}$, which describes the average rate of radial transport, is given by

$$
D_{L L}=D_{L L}^{E}+D_{L L}^{B},
$$

where $D_{L L}^{E}$ and $D_{L L}^{B}$ are the diffusion coefficients due to symmetric interactions of electrons with electrostatic and electromagnetic variations, respectively [Fälthammar, 1965; Cornwall, 1968].

[33] There are many studies that have examined the mathematical form of $D_{L L}^{E}$ and/or $D_{L L}^{B}$ [e.g., Fälthammar, 1965; Schulz and Lanzerotti, 1974; Elkington et al., 1999, 2003; Brizard and Chan, 2004; Brautigam et al., 2005; Perry et al., 2005; Fei et al., 2006]. For this study, the analytic forms presented by Fei et al. [2006] are used to estimate radial diffusion. These equations can be written as

$$
D_{L L}^{E}=\frac{1}{8 B_{s}^{2} R_{E}^{2}} \cdot L^{6} \cdot \sum_{m} P_{m}^{E}
$$

and

$$
D_{L L}^{B}=\frac{\mu^{2}}{8 q^{2} \gamma^{2} B_{s}^{2} R_{E}^{4}} \cdot L^{4} \cdot \sum_{m} m^{2} P_{m}^{B}
$$

Here, $B_{S}$ is the equatorial magnetic field at Earth's surface, $R_{E}$ is Earths radius, $L$ the McIlwain $L$ value, $\gamma$ is the relativistic Lorentz factor, $\mu$ is the relativistic first adiabatic invariant (see equation (1)), $m$ is the azimuthal wave mode number, $q$ is the electron charge and $P_{m}^{E}$ and $P_{m}^{B}$ are the power spectral densities of the wave azimuthal electric field and the compressional wave magnetic field, respectively; both evaluated at $\omega=m\left\langle\omega_{d}\right\rangle$. Equation (5) agrees exactly with Fälthammar [1965], while equation (6) also agrees with Fälthammar [1965] when taking the nonrelativistic limit and assuming $m=1$ [Fei et al., 2006]. Inverting equations (4)-(6) provides estimates of the time scale $\left(\tau_{L L}\right)$ for an electron to diffuse $1 R_{E}$.

\subsection{Magnetic and Electric Power Spectral Densities Versus $L$ Value}

[34] On 25 June 2008 at 00 UT, THM-A was located in the afternoon sector near the magnetopause and moving inbound reaching just below geosynchronous altitudes by $\sim 05$ UT. Both $P_{m}^{E}$ and $P_{m}^{B}$ in equations (5) and (6) were calculated using the method described by Loto'aniu et al. [2006a], where the power spectral density is calculated by summing the power spectrum over a spectral width, $\delta f$, and then dividing the result by $\delta f$ to create a spectral density. The THM-A Ey_FAC and Bz_FAC data (see Figure 6) over $\sim 00-05$ UT were used to calculate $P_{m}^{E}$ and $P_{m}^{B}$ using the Fast Fourier Transformation with a 600 point $(30 \mathrm{~min})$ FFT, a 60 point $(3 \mathrm{~min})$ slide time or time resolution, and taking $\delta f=3 \mathrm{mHz}$ with spectral power summed over $2-5 \mathrm{mHz}$. Assuming that $\mathrm{Bz}$ FAC corresponds to the compressional magnetic field and Ey_FAC to the azimuthal electric field, the radial diffusion coefficients were calculated as a function of $L$ value. The power spectral densities $\left(P_{m}^{E}\right.$ and $\left.P_{m}^{B}\right)$ and radial diffusion coefficient time scales (in minutes) are shown in Figure 11, assuming a dipole field and the T96 field model [Tsyganenko, 1995] to determine $L$ values.

[35] For $L$ value calculations, the magnetic field values at the equator, $B_{e q}$, were estimated by mapping the THMA locations to the magnetic equator of the field model used. $L$ values are then calculated using $L=\left(B_{S} / B\right)^{1 / 3}$, where $B_{s}$ is the equatorial magnetic field at Earth's surface.

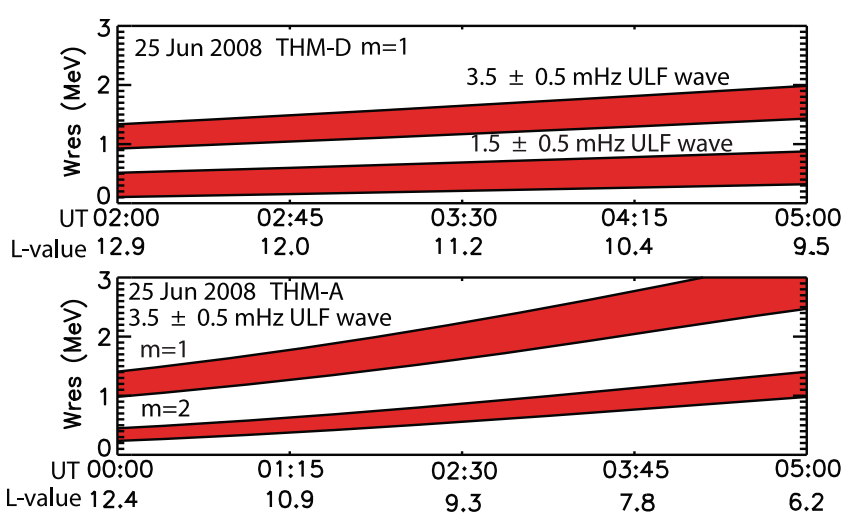

Figure 10. Resonant energies $W_{\text {res }}$ of electrons interacting with the observed ULF waves at THM-D and THM-A locations using equation (3), $90^{\circ}$ pitch angle electrons, and the observed ULF wave spectral properties. 

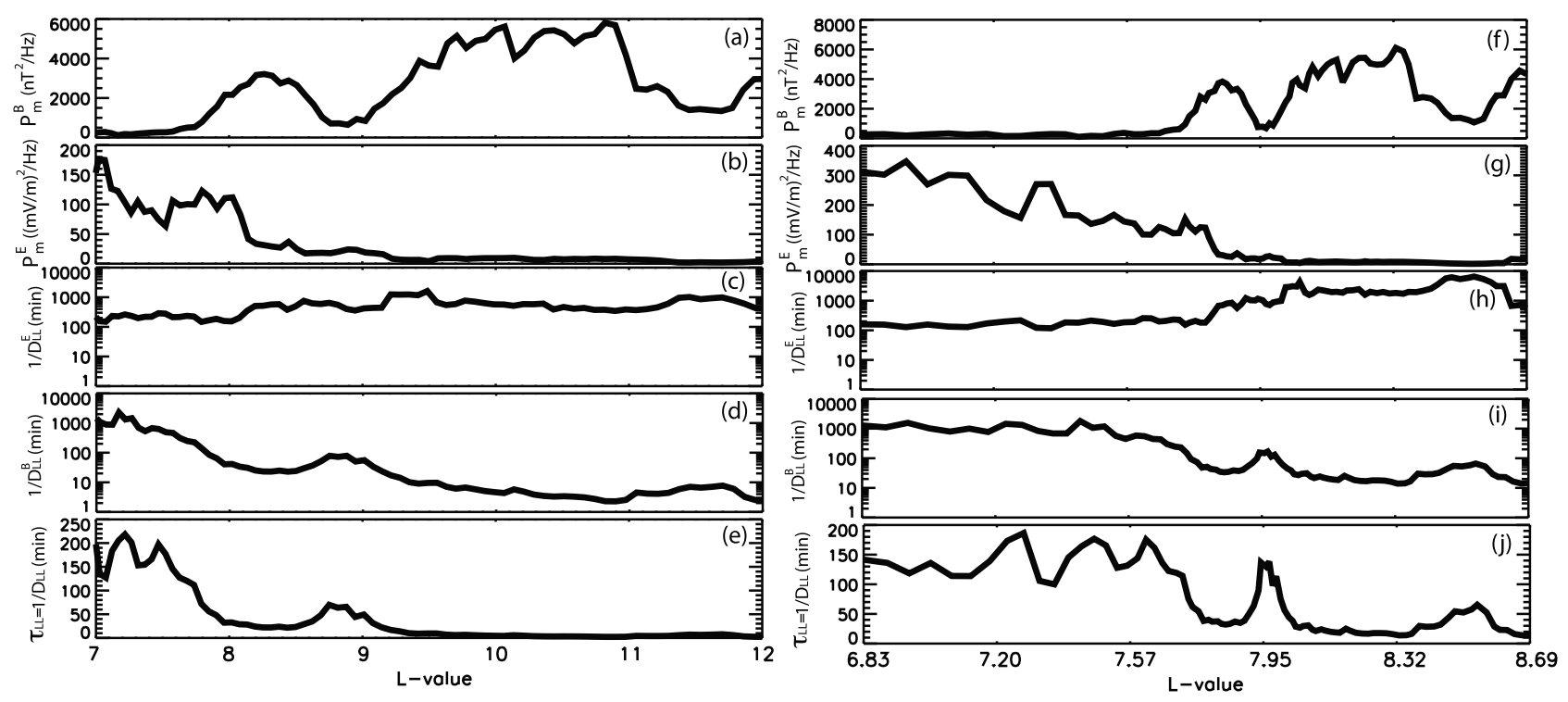

Figure 11. The power spectral densities and corresponding radial diffusion coefficients over the $L$ range that THM-A traversed over $\sim 00-05$ UT on 25 June 2008. Using a dipole field to estimate $L$ values, (a) $P_{m}^{B}$ and (b) $P_{m}^{E}$ were calculated using THM-A electric and magnetic data. (c-e) The diffusion time scales or inverses of the $D_{L L}^{E}, D_{L L}^{B}$, and $D_{L L}$ in minutes. (f-j) Same as in Figures $11 \mathrm{a}-11 \mathrm{e}$, except using the T96 field model to estimate $L$ values.

In Figure 11, the $L$ value ranges are significantly different when calculated using a dipole field compared to using the T96 field model. The time spent by THM-A at each $L$ is also different, for example, the power with $L$ based on the T96 model is stretched in the first half of Figure 11 and compressed in the second half compared to the dipole results. Concentrating on results that use a dipole field (Figures $11 \mathrm{a}-11 \mathrm{e}$ ), the $P_{m}^{B}$ has two regions that show peaks in power; $L=8-9$ and $L=9-11$, while minimal power occurred near geosynchronous orbit. Except for a peak between $L \sim 7.5-8, P_{m}^{E}$ generally decreases with increasing $L$.

\subsection{Radial Diffusion Time Scales}

[36] In Figure 11, all values were calculated at the beginning of the FFT interval, with time resolutions of 3 min corresponding to the dynamic FFT slide time used to estimate magnetic and electric power spectral densities. $D_{L L}^{B}$ was estimated assuming $\mu \sim 1800 \mathrm{MeV} / \mathrm{G}$. Results show that the diffusion time, $\tau_{L L}=1 / D_{L L}$, for $\sim 1 \mathrm{MeV}$ electrons to diffuse $\sim 1 R_{E}$ from near geosynchronous orbit was about 140-160 min ( 2.3-2.7 h), while near the magnetopause diffusion times were much faster; below 20 min when using T96, and $\sim 1$ min using a dipole model. The diffusion time near geosynchronous orbit is consistent with the assumed nonadiabatic loss time of $1-4 \mathrm{~h}$ presented in section 3.5 . The contribution to $D_{L L}$ at $L=6.6$ (dipole) from $D_{L L}^{E}$ was over $90 \%$, while at the magnetopause the electric diffusion term contributes less than $2 \%$ to the overall radial diffusion rate. These results are discussed in section 6 .

\section{Discussion}

[37] The results in this study provide evidence for the mechanism presented in Figure 1, whereby relativistic electron loss through the magnetopause is enhanced due to a combination of magnetospheric compression and ULF wave-particle interaction resulting in enhanced outward diffusion. In Figure 1a, the radiation belt conditions just before the event assumes phase space density peaking for 1-2 MeV electrons near geosynchronous altitude. This assumption was based on results from recent studies [Turner and Li, 2008; Chen et al., 2005, 2006, 2007; Iles et al., 2006]; however, an initial local peak is not required for the mechanism presented.

[38] The pressure enhancements over 20-24 UT on 24 June compresses the magnetosphere resulting in electron loss due to magnetopause shadowing across all energies. It is well known that magnetospheric compression can enhance losses in the outer zone [e.g., Kim et al., 2008] and this explains at least part of the flux losses observed by GOES and THEMIS on 25 June. However, Green et al. [2004] studied 52 electron flux depletion events at geostationary orbit and concluded that magnetopause encounters were not responsible for the permanent loss of electrons. Figure 4 shows relativistic losses observed across the entire outer radiation belt. Although the magnetosphere compresses to within $2 R_{E}$ of geosynchronous orbit on 25 June, it is unlikely that losses across the entire outer zone radiation belt can be explained by simple magnetopause shadowing.

[39] The increased solar wind speed that was sustained over several days (Figure 2) indicates an association with a corotating interaction region (CIR) [Gosling, 1996]. Studies suggest CIRs are more efficient than coronal mass ejections (CMEs) at transferring energy into the magnetosphere over a sustained period [Turner et al., 2006]. The efficiency by which CIRs transfer energy into the magnetosphere may play a role in explaining the significant amplitudes of the Pc 5 ULF waves observed by the THEMIS spacecraft. 
[40] The moderate ring current (Sym-H $>-40 \mathrm{nT}$ ) during the event suggests that relativistic losses were unlikely to be related to electron drift orbits expanding and intersecting with the magnetopause as a result of diamagnetic influences from the ring current (Dst effect), as was the case in the Ukhorskiy et al. [2006] study. Kp was also low $(<3)$ during the observed $1-4 \mathrm{~h}$ that relativistic losses occurred. Previous studies have often used empirically derived diffusion coefficients that incorporate $\mathrm{Kp}$ as a proxy for wave activity [e.g., Brautigam et al., 2005; Shprits et al., 2006]. The results of Shprits et al. [2006], for example, suggest that Kp must be around 5 or higher for significant outward diffusion and depletion of outer zone radiation belt relativistic electrons. Hence, the use of Kp as a proxy by Shprits et al. does not suitably cover the scenario presented here. Moreover, in order for wave-particle interactions to produce significant radial diffusion, waves must have frequency, polarization and azimuthal mode numbers that satisfy drift resonance conditions [e.g., Loto'aniu et al., 2006a]. The use of proxies do not guarantee that the waves possess the correct wave spectral properties, or that they were in the correct location and possessed sufficient wave power over the appropriate times.

[41] Properties of the ULF waves were found to be in good agreement with the requirements for strong waveparticle drift resonant interactions [e.g., Loto'aniu et al., 2006a]. The minimum drift resonance energies, $W_{\text {res }}$, were estimated assuming a dipole field. However, the ULF power spectra shown in Figure 6 covers a broad range of frequencies and implies that multiple $m$ numbers existed, while the minimum resonant energy curves shown in Figure 10 indicate a continuum of resonant energies from $100 \mathrm{~s}$ of $\mathrm{keV}$ to $\mathrm{MeV}$ electrons. Hence, it is likely that the resonance condition would be satisfied within this energy range, irrespective of the field model used to calculate $W_{\text {res }}$. As electrons diffuse outward, in response to the magnetospheric compression, they encounter ULF waves. Wave-particle interactions with multiple wave modes affect electrons over a broad range of energies.

[42] In Figure 11, the power spectral density of the electric field, $P_{m}^{E}$, generally decreased with increasing $L$, while for the magnetic field power spectral density, $P_{m}^{B}$, two local power peaks dominate the curves, while power tends to increase toward the magnetopause compared to geosynchronous orbit. Spacecraft observations have been used to study the distribution of ULF waves in the magnetosphere by a number of authors [e.g., Anderson et al., 1990; Lessard et al., 1999; Brautigam et al., 2005; Liu et al., 2009]. For example, Brautigam et al. [2005] found empirical forms for the radial diffusion coefficients based on the parameterization of statistical wave power in terms of $L$ and $\mathrm{Kp}$ using CRRES data. Brautigam et al. determined the electric field power spectral density as a function of $L$ and $\mathrm{Kp}$ over the frequency range $0.2-15.9 \mathrm{mHz}$, with the power at different frequencies determined by fitting spectra to power laws. In the range $L=3-7$, they found that wave electric power generally increases with increasing $L$. More recently, a statistical study by Liu et al. [2009] using THEMIS ULF wave observations also found that Pc 5 wave electric field power increases with increasing $L$. In that study, the $L$ range includes the $L$ values covered here. However, the Brautigam et al. study also found that as much as $16 \%$ of the individual power spectra differ from the model power spectra by a factor of $\sim 10$. In other words, the actual wave power spectrum can vary significantly from its power law fit. Since the wave power is such a critical part of radiation belt physics, more complete understanding of radiation belt dynamics requires event based studies that utilize the actual observed wave power as in this study.

[43] In Figures 11a and 11b, the overlapping period of enhanced magnetic and electric power at $L \sim 8$ might indicate electromagnetic activity, for example, a ULF wave due to field line resonance (FLR). Enhanced electrostatic variations around geosynchronous orbit, on the other hand, may suggest significant variations in the convective electric field. The results imply that the ULF pulsations observed by the THEMIS probes over the time interval 00-05 UT on 25 June originate from a mixture of different sources. However, differentiating contributions from each source to the total wave amplitude is beyond the scope of this study.

[44] Interpretation of the diffusion coefficients shown in Figure 11 assumes that THM-A observations of ULF wave power are time independent over the interval 00-05 UT. Although, THM-D and -E on average measure larger amplitude waves near the magnetopause than THM-A, qualitative comparisons between the three probes along the entire $5 \mathrm{~h}$ interval indicate that differences in observed wave amplitudes do not change the results significantly and reinforce the conclusions. Hence, it is reasonable to assume that the $L$ dependency of both $P_{m}^{B}$ and $P_{m}^{E}$ were time independent over the $5 \mathrm{~h}$ of observation used to estimate diffusion coefficients.

[45] The diffusion times, $\tau_{L L}=1 / D_{L L}$, shown in Figures 11e and $11 \mathrm{j}$ are consistent with the mechanism presented in section 2. Near geosynchronous orbit, the rate of diffusion of 2.3-2.7 h (140-160 min) is in good agreement with the assumed nonadiabatic loss time of 1-4 h base on observations presented in section 3.5. Close to the magnetopause, the rate of radial diffusion is high ( $<20 \mathrm{~min})$ indicating rapid loss and enhanced outward negative phase space density as the electrons approach the magnetopause.

[46] Although the rates of radial diffusion are consistent with observations, the assumptions inherent in the $D_{L L}$ equation (4) imply that the estimated diffusion rates are lower limits. For example, the diffusion model used assumes only symmetric interactions. Adding the asymmetric $(m \pm 1)$ resonance terms would mean even faster diffusion times [Elkington et al., 2003]. Since there are always opposing mechanisms in play within the magnetosphere, the rate of outward diffusion needs to be fast enough to overcome any simultaneous acceleration processes.

[47] The assumed nonadiabatic loss time of $1-4 \mathrm{~h}$ could be disputed as there is no way to truly separate the adiabatic and nonadiabatic responses using limited observations. As stated in the introduction, the phase space density was not calculated because the particle instruments onboard the THEMIS probes are still undergoing cross-calibration. Hence, it would be difficult to justify the accuracy of phase space density versus $L$ estimated using THEMIS or using a combination of THEMIS and GOES satellites. It is therefore arguable that in this paper estimating the phase space density for the event on 25 June would not improve our confidence in the mechanism presented in section 2. 
[48] The ULF waves used to estimate the diffusion times shown in Figure 11 were observed in the afternoon and dusk sectors. However, ground-based magnetometer observations by the INTERMAGNET network (not shown here) indicate that the ULF waves observed on 25 June 2008, occurred across a wide region of local times. Hence, outward diffusion was not necessarily restricted to the local sectors were THEMIS observed ULF waves. Furthermore, the rate of diffusion will vary with local time as wave power varies azimuthally. During the loss event, solar wind dynamic pressure was enhanced, while the interplanetary magnetic field tended to be southward (Figure 2). Under such conditions, the dayside magnetospheric compression enhances the day-night magnetic asymmetry [e.g., Ohtani et al., 2009]. As a consequence, the expanded number of open electron drift paths most likely encounter the magnetopause at local noon, hence, significant losses may occur at local noon.

[49] The rates of radial diffusion were estimated using different field models to calculate $L$ in order to gauge the affects of changing $L$ on the overall diffusion rates. The diffusion rates were also calculated (not shown) using the TS05 (or TS04) model [Tsyganenko and Sitnov, 2005], with results similar to the T96 results. Although, changing the field model changes the $L$ covered by THM-A over the 00-05 UT interval, it does not significantly change the diffusion rates near geosynchronous orbit or near the magnetopause. An attempt to calculate the Roederer $L$ [Roederer, 1970], $L^{*}$, using the ONERA codes [Boscher et al., 2004] failed. This was possibly due to THM-A traversing the outer magnetosphere where closed drift paths are difficult to estimate. The similarities between results shown in Figure 11e and $11 \mathrm{j}$, even though they have different $L$ ranges, suggests that as long as $L^{*}$ falls within the $L$ range shown in Figure $11 \mathrm{e}$, the diffusion rates calculated using $L^{*}$ should not be significantly different. Huang et al. [2008] estimated $L^{*}$ for a moderate storm to study the effect of using different field models. They found that at geosynchronous orbit, where $L=L^{*}=6.6$ for a dipole field, $L^{*}$ was 6.0 when using TS05. Near the magnetopause, field line stretching is more pronounced at the flanks and nightside, hence, the actual value of $L^{*}$ near the magnetopause in Figure 11e is likely lower than $L=12$. These differences are not enough to significantly change the results.

[50] As stated in section 5, the form of $D_{L L}^{E}$ given in equation (5) agrees exactly with Fälthammar [1965]. The $L$ dependence of the magnetic diffusion coefficient, $D_{L L}^{B}$, is more complicated since $\gamma$ also depends on $L$ [Elkington, 2006; Fei et al., 2006]. In the ultra-relativistic limit $\gamma^{2} \propto L^{-3}$, so that for radiation belt electrons $L^{4} / \gamma^{2}$ is approximately proportional to $L^{7}$, not including the $L$ dependence implicit in $P_{m}^{B}$ [Elkington, 2006]. Equation (6) does not exhibit the same explicit $L^{10}$ as Fälthammar [1965] because its relativistic, does not assume $\mathrm{m}=1$ and does not include an explicit $L$ dependency for the perturbing fields $[\mathrm{Fe} i$ et al., 2006]. When wave power is considered, the general $L$ dependency of $D_{L L}$ is more complicated, particularly when $L$ was calculated using the more realistic field models. Due to local peaks in power, it was not possible to accurately fit observations to power laws for either $D_{L L}^{E}$ or $D_{L L}^{B}$ shown in Figure 11. The statistical study of Brautigam et al. [2005] suggests that when using the wave electric field, the general $L$ dependency of $D_{L L}$ may be steeper than $L^{6}$ in the outer radiation belt, although, the $L$ range used by Brautigam et al. is below the $L$ range in this study.

[51] Equation (5) does not include the energy correction factor [Cornwall, 1968; Brautigam and Albert, 2000], which can modify the overall $L$ dependency of $D_{L L}^{E}$. The spectral density of the electric field power was calculated using the method described by Loto'aniu et al. [2006a], where the power spectrum is summed over the signal spectral width (in this case, over $2-5 \mathrm{mHz}$ ) and then divided by that spectral width. This produces a flat spectral density that is frequency-independent within the spectral range summed $(2-5 \mathrm{mHz})$. For ULF electric wave oscillations that produce flat frequency spectra, the $D_{L L}^{E}$ retains the $L$ dependency in equation (5) [Perry et al., 2005].

[52] The use of equations (5) and (6) implicitly assumes that $P_{m}^{E}$ is derived from purely electrostatic variations and $P_{m}^{B}$ due to electromagnetic variations. However, because the THEMIS probes observe perturbations that originate from a mixture of different sources it is hard to differentiate contributions from each source to the total wave amplitude. As a consequence, some electrostatic diffusion be may counted in the magnetic diffusion coefficient. The amount of over counting, however, is very difficult to estimate. Near geosynchronous altitude, the contribution to $D_{L L}$ from $D_{L L}^{E}$ is over $90 \%$, while at the magnetopause it was less than $2 \%$. This corresponds to the wave power being predominately electrostatic near geosynchronous orbit, while toward the magnetopause it was observed mainly in the magnetic fields. Over counting of the $D_{L L}^{E}$ may be more of an issue at $L \sim 8-9$ (dipole), where $P_{m}^{E}$ and $P_{m}^{B}$ are both enhanced indicating a possible FLR centered at that region. However, at $L \sim 8$ (dipole) the diffusion time due to $D_{L L}^{B}$ alone is still fast $(<1 \mathrm{~h})$.

[53] We believe that the multispacecraft observations presented here are the first to demonstrate the loss of outer radiation belt relativistic electrons to the magnetopause in response to ULF waves. Although previous studies [e.g., Shprits et al., 2006] have used proxies for ULF waves, this is the first time that the observed ULF wave spectral properties were shown to be consistent with this mechanism during a relativistic electron loss event. An obvious question is whether or not this scenario is typical during outer zone radiation belt depletions. Iles et al. [2006] found that it is possible to have inward radial diffusion at low energies and outward radial diffusion at higher energies. Therefore, it may be possible for ULF waves to contribute to both acceleration and loss. The phase space density gradient, wave spectral properties and location within the magnetosphere are all important parameters to determine the role of ULF waves in radiation belt dynamics. The THEMIS spacecraft provides a rare, but excellent opportunity to help answer this question and data from THEMIS will be used for future studies.

\section{Conclusion}

[54] In this study the radial diffusion time scales for relativistic electrons drift resonating with Pc 5 ULF waves were estimated from 00-05 UT on 25 June 2008, when THEMIS and GOES spacecraft observed relativistic electron losses in the outer zone radiation belt. Adiabatic losses were unlikely to account for the overall loss of $0.65 \mathrm{MeV}$ 
outer zone radiation belt electrons measured by the THM-E solid state telescope instrument. A scenario was presented to explain the observations whereby a combination of a magnetospheric compression and ULF waves near the magnetopause enhances losses through the magnetopause. Magnetopause shadowing from the compression results in a negative radial phase space density gradient near and beyond geosynchronous altitudes for constant first adiabatic invariant, causing outer zone radiation belt electrons to diffuse outward toward the magnetopause while interacting with ULF waves. GOES data suggest that nonadiabatic loss on 25 June occurred over a time scale of 1-4 h. At the same time, THEMIS spacecraft and CARISMA ground magnetometers observed intense ULF waves extending from the magnetopause to geosynchronous orbit. Estimates of resonant energies suggest that outward diffusing electrons, due to the magnetospheric compression, may interact with the observed ULF waves. The radial diffusion rates were estimated using the THEMIS observed ULF wave azimuthal electric field and the compressional magnetic field. An indication of the ULF wave radial extent and azimuthal wave number were provided by the ground-based CARISMA magnetometer network, while observations by INTERMAGNET magnetometer network suggest waves were observed across a wide range of local times. The combined electric and magnetic diffusion rates indicate that the diffusion time was in good agreement with the observed time for the nonadiabatic flux decreases. This study demonstrates that a combination of magnetospheric compression and ULF wave-particle drift resonant interaction may result in enhanced outward radial diffusion and produce losses at time scales sufficient to explain rapid nonadiabatic relativistic loss from the outer radiation belt. Future studies of radiation belt losses should consider the relative importance of ULF waves in the loss process as well as acceleration.

[55] Acknowledgments. This study was funded by the NOAA GOES-R risk reduction program. Work at IGPP-UCLA was supported by NASA THEMIS grant NAS5-02099. We thank Terry Onsager, Janet Green, Juan Rodriguez, Jonathan Rae, Louis Ozeke, and Josh Rigler for helpful discussions. We also thank Terry Onsager for his work in preparing the GOES energetic particle data. We thank K. H. Glassmeier, U. Auster, and $\mathrm{W}$. Baumjohann for the use of FGM data provided under the lead of the Technical University of Braunschweig and with financial support through the German Ministry for Economy and Technology and the German Center for Aviation and Space (DLR) under contract 50 OC 0302. CARSIMA is the magnetometer element of the Canadian Geospace Monitoring (CGSM) project and funded by the CSA.

[56] Masaki Fujimoto thanks the reviewers for their assistance in evaluating this manuscript.

\section{References}

Albert, J. M. (2005), Evaluation of quasi-linear diffusion coefficients for whistler mode waves in a plasma with arbitrary density ratio, J. Geophys. Res., 110, A03218, doi:10.1029/2004JA010844.

Anderson, B. J., M. J. Engebretson, S. P. Rounds, L. J. Zanetti, and T. A. Potemra (1990), A statistical study of Pc 3-5 pulsations observed by the AMPTE/CCE magnetic field experiment: 1. Occurrence distributions, J. Geophys. Res., 95(A7), 10,495-10,523, doi:10.1029/JA095iA07p10495. Angelopoulos, V. (2008), The THEMIS Mission, Space Sci. Rev., 141, 5-34, doi:10.1007/s11214-008-9336-1.

Auster, H. U., et al. (2008), The THEMIS fluxgate magnetometer, Space Sci. Rev., 141, 235-264.

Bonnell, J., F. Mozer, G. Delory, A. Hull, R. Ergun, C. Cully, V. Angelopoulos, and P. Harvey (2008), The electric field instrument (EFI) for THEMIS, Space Sci. Rev., 141, 303-341.
Boscher, D., S. Bourdarie, P. O’Brien, and T. Guild (2004), Onera-desp library ver. 4.2, technical report, ONERA-DESP, Toulouse-France.

Brautigam, D. H., and J. M. Albert (2000), Radial diffusion analysis of outer radiation belt electrons during October 9, 1990, magnetic storm, J. Geophys. Res., 105(A1), 291-309, doi:10.1029/1999JA900344.

Brautigam, D. H., G. P. Ginet, J. M. Albert, J. R. Wygant, D. E. Rowland, A. Ling, and J. Bass (2005), CRRES electric field power spectra and radial diffusion coefficients, J. Geophys. Res., 110, A02214, doi:10.1029/ 2004JA010612.

Brizard, A. J., and A. A. Chan (2004), Relativistic quasi-linear diffusion in axisymmetric magnetic geometry for arbitrary-frequency electromagnetic fluctuations, Phys. Plasmas, 11, 4220-4229.

Chen, Y. R., H. W. Friedel, G. D. Reeves, T. G. Onsager, and M. F. Thomsen (2005), Multisatellite determination of the relativistic electron phase space density at geosynchronous orbit: Methodology and results during geomagnetically quiet times, J. Geophys. Res., 110, A10210, doi:10.1029/ 2004JA010895.

Chen, Y. R., H. W. Friedel, and G. D. Reeves (2006), Phase space density distributions of energetic electrons in the outer radiation belt during two geospace environment modeling inner magnetosphere/storms selected storms, J. Geophys. Res., 111, A11S04, doi:10.1029/2006JA011703.

Chen, Y. R., H. W. Friedel, G. D. Reeves, T. E. Cayton, and R. Christensen (2007), Multisatellite determination of the relativistic electron phase space density at geosynchronous orbit: An integrated investigation during geomagnetic storm times, J. Geophys. Res., 112, A11214, doi:10.1029/ 2007JA012314.

Cornwall, J. (1968), Diffusion processes influenced by conjugate-point wave phenomena, Radio Sci., 3, 740.

Elkington, S. R. (2006), A review of ULF interactions with radiation belt electrons, in Magnetospheric ULF Waves: Synthesis and New Directions, Geophys. Monogr. Ser., vol. 169, edited by T. Kazue, P. J. Chi, and R. E. Denton, pp. 177-194, AGU, Washington, D. C.

Elkington, S. R., M. K. Hudson, and A. A. Chan (1999), Acceleration of relativistic electrons via drift-resonant interaction with toroidal-mode Pc-5 ULF oscillations, Geophys. Res. Lett., 26(21), 3273-3276, doi:10.1029/1999GL003659.

Elkington, S. R., M. K. Hudson, and A. A. Chan (2003), Resonant acceleration and diffusion of outer zone electrons in an asymmetric geomagnetic field, J. Geophys. Res., 108(A3), 1116, doi:10.1029/ 2001JA009202.

Fairfield, D. H. (1971), Average and unusual locations of the Earth's magnetopause and bow shock, J. Geophys. Res., 76(28), 6700-6716, doi:10.1029/JA076i028p06700.

Fälthammar, C.-G. (1965), Effects of time-dependent electric fields on geomagnetically trapped radiation, J. Geophys. Res., 70(11), 2503-2516, doi:10.1029/JZ070i011p02503.

Fei, Y., A. A. Chan, S. R. Elkington, and M. J. Wiltberger (2006), Radial diffusion and MHD particle simulations of relativistic electron transport by ULF waves in the September 1998 storm, J. Geophys. Res., 111, A12209, doi:10.1029/2005JA011211.

Gosling, J. T. (1996), Corotating and transient solar wind flows in three dimensions, Ann. Rev. Astron. Astrophys., 34, 35-73.

Green, J. C., and M. G. Kivelson (2004), Relativistic electrons in the outer radiation belt: Differentiating between acceleration mechanisms, J. Geophys. Res., 109, A03213, doi:10.1029/2003JA010153.

Green, J. C., T. G. Onsager, T. P. O’Brien, and D. N. Baker (2004), Testing loss mechanisms capable of rapidly depleting relativistic electron flux in the Earth's outer radiation, J. Geophys. Res., 109, A12211, doi:10.1029/ 2004JA010579.

Hamlin, D. A., R. Karplus, R. C. Vik, and K. M. Watson (1961), Mirror and azimuthal drift frequencies for geomagnetically trapped particles, J. Geophys. Res., 66(1), 1-4, doi:10.1029/JZ066i001p00001.

Huang, C.-L., H. E. Spence, H. J. Singer, and N. A. Tsyganenko (2008), A quantitative assessment of empirical magnetic field models at geosynchronous orbit during magnetic storms, J. Geophys. Res., 113, A04208, doi:10.1029/2007JA012623.

Hughes, W. J. (1974), Mirror and azimuthal drift frequencies for geomagnetically trapped particles, Planet. Space Sci., 22, 1157-1172.

Iles, R. H. A., N. P. Meredith, A. N. Fazakerley, and R. B. Horne (2006), Phase space density analysis of the outer radiation belt energetic electron dynamics, J. Geophys. Res., 111, A03204, doi:10.1029/2005JA011206.

Kim, H.-J., and A. A. Chan (1997), Fully adiabatic changes in storm time relativistic electron fluxes, J. Geophys. Res., 102(A10), 22,107-22,116, doi:10.1029/97JA01814.

Kim, K. C., D.-Y. Lee, H.-J. Kim, L. R. Lyons, E. S. Lee, M. K. Öztürk, and C. R. Choi (2008), Numerical calculations of relativistic electron drift loss effect, J. Geophys. Res., 113, A09212, doi:10.1029/2007JA013011. 
Lessard, M. R., M. K. Hudson, and H. Luhr (1999), A statistical study of Pc3-Pc5 magnetic pulsations observed by the AMPTE/Ion Release Module satellite, J. Geophys. Res., 104, 4523, doi:10.1029/1998JA900116.

Li, X., D. N. Baker, S. G. Kanekal, M. Looper, and M. Temerin (2001), Long term measurements of radiation belts by SAMPEX and their variations, Geophys. Res. Lett., 28(20), 3827-3830, doi:10.1029/2001GL013586.

Li, X. L., and M. A. Temerin (2001), The electron radiation belt, Space Sci. Rev., 95, 569-580.

Liu, W., T. E. Sarris, X. Li, S. R. Elkington, R. Ergun, V. Angelopoulos, J. Bonnell, and K. H. Glassmeier (2009), Electric and magnetic field observations of Pc4 and Pc5 pulsations in the inner magnetosphere: A statistical study, J. Geophys. Res., 114, A12206, doi:10.1029/ 2009JA014243

Loto'aniu, T. M., I. R. Mann, L. G. Ozeke, A. A. Chan, Z. C. Dent, and D. K. Milling (2006a), Radial diffusion of relativistic electrons into the radiation belt slot region during the 2003 Halloween geomagnetic storms, J. Geophys. Res., 111, A04218, doi:10.1029/2005JA011355.

Loto'aniu, T. M., R. M. Thorne, B. J. Fraser, and D. Summers (2006b), Estimating relativistic electron pitch angle scattering rates using properties of the electromagnetic ion cyclotron wave spectrum, J. Geophys. Res., 111, A04220, doi:10.1029/2005JA011452.

Mann, I. R., et al. (2009), The upgraded CARISMA magnetometer array in the THEMIS era, in The THEMIS Mission, edited by J. L. Burch and V. Angelopoulos, pp. 413-451, Springer, New York

Meredith, N. P., R. M. Thorne, R. B. Horne, D. Summers, B. J. Fraser, and R. R. Anderson (2003), Statistical analysis of relativistic electron energies for cyclotron resonance with EMIC waves observed on CRRES, J. Geophys. Res., 108(A6), 1250, doi:10.1029/2002JA009700.

Millan, R. M., and R. M. Thorne (2007), Review of radiation belt relativistic electron losses, J. Atmos. Sol. Terr. Phys., 69, 362-377.

Millan, R. M., R. P. Lin, D. M. Smith, and M. P. McCarthy (2007), Observation of relativistic electron precipitation during a rapid decrease of trapped relativistic electron flux, Geophys. Res. Lett., 34, L10101, doi:10.1029/2006GL028653.

Miyoshi, Y., A. Morioka, T. Obara, H. Misawa, T. Nagai, and Y. Kasahara (2003), Rebuilding process of the outer radiation belt during the 3 November 1993 magnetic storm: NOAA and Exos-D observations, J. Geophys. Res., 108(A1), 1004, doi:10.1029/2001JA007542.

Ohtani, S., Y. Miyoshi, H. J. Singer, and J. M. Weygand (2009), On the loss of relativistic electrons at geosynchronous altitude: Its dependence on magnetic configurations and external conditions, J. Geophys. Res., 114, A01202, doi:10.1029/2008JA013391.

Olson, J., and G. Rostoker (1978), Longitudinal phase variations of Pc4-5 micropulsations, J. Geophys. Res., 83(A6), 2481-2488, doi:10.1029/ JA083iA06p02481.

Ozeke, L. G., and I. R. Mann (2008), Energization of radiation belt electrons by ring current ion driven ulf waves, J. Geophys. Res., 113, A02201, doi:10.1029/2007JA012468.

Perry, K. L., M. K. Hudson, and S. R. Elkington (2005), Incorporating spectral characteristics of $\mathrm{Pc} 5$ waves into three-dimensional radiation belt modeling and the diffusion of relativistic electrons, J. Geophys. Res. 110, A03215, doi:10.1029/2004JA010760.

Reeves, G. D., K. L. McAdams, R. H. W. Friedel, and T. P. O'Brien (2003), Acceleration and loss of relativistic electrons during geomagnetic storms, Geophys. Res. Lett., 30(10), 1529, doi:10.1029/2002GL016513.

Roederer, J. G. (1970), Dynamics of Geomagnetically Trapped Radiation, vol. 2, Springer, New York.

Roelof, E. C., and D. G. Sibeck (1993), Magnetopause shape as a bivariate function of interplanetary magnetic field $B_{z}$ and solar wind dynamic pressure, J. Geophys. Res., 98(A12), 21,421-21,450.

Schulz, M., and L. J. Lanzerotti (1974), Particle Diffusion in the Radiation Belts, Springer, New York.

Selesnick, R. S. (2006), Source and loss rates of radiation belt relativistic electrons during magnetic storms, J. Geophys. Res., 111, A04210, doi:10.1029/2005JA011473.

Shprits, Y. Y., R. M. Thorne, R. Friedel, G. D. Reeves, J. Fennell, D. N. Baker, and S. G. Kanekal (2006), Outward radial diffusion driven by losses at magnetopause, J. Geophys. Res., 111, A11214, doi:10.1029/ 2006JA011657.
Shue, J.-H., et al. (1998), Magnetopause location under extreme solar wind conditions, J. Geophys. Res., 103(A8), 17,691-17,700, doi:10.1029/ 98JA01103

Sibeck, D., and V. Angelopoulos (2008), THEMIS science objectives and mission phases, Space Sci. Rev., 141, 35-59, doi:10.1007/s11214-0089393-5.

Summers, D., and R. M. Thorne (2003), Relativistic electron pitch angle scattering by electromagnetic ion cyclotron waves during geomagnetic storms, J. Geophys. Res., 108(A4), 1143, doi:10.1029/2002JA009489.

Summers, D., B. Ni, and N. P. Meredith (2007a), Timescales for radiation belt electron acceleration and loss due to resonant wave-particle interactions: 2. Evaluation for VLF chorus, ELF hiss, and electromagnetic ion cyclotron waves, J. Geophys. Res., 112, A04207, doi:10.1029/ 2006JA011993.

Summers, D., B. Ni, and N. P. Meredith (2007b), Timescales for radiation belt electron acceleration and loss due to resonant wave-particle interactions: 1. Theory, J. Geophys. Res., 112, A04206, doi:10.1029/2006JA011801.

Summers, D., B. Ni, N. P. Meredith, R. B. Horne, R. M. Thorne, M. B. Moldwin, and R. R. Anderson (2008), Electron scattering by whistler mode ELF hiss in plasmaspheric plumes, J. Geophys. Res., 113 A04219, doi:10.1029/2007JA012678.

Tsyganenko, N. A. (1995), Modeling the Earth's magnetospheric magnetic field confined within a realistic magnetopause, J. Geophys. Res., 100(A4), 5599-5612, doi:10.1029/94JA03193.

Tsyganenko, N. A., and M. I. Sitnov (2005), Modeling the dynamics of the inner magnetosphere during strong geomagnetic storms, J. Geophys. Res., 110, A03208, doi:10.1029/2004JA010798.

Turner, D., and X. Li (2008), Radial gradients of phase space density of the outer radiation belt electrons prior to sudden solar wind pressure enhancements, Geophys. Res. Lett., 35, L18101, doi:10.1029/ 2008GL034866.

Turner, N. E., E. J. Mitchell, D. J. Knipp, and B. A. Emery (2006), Energetics of magnetic storms driven by corotating interaction regions: A study of geoeffectiveness, in Recurrent Magnetic Storms: Corotating Solar Wind Streams, Geophys. Monogr. Ser., vol. 167, edited by B. Tsurutani et al., p. 113-124, AGU, Washington, D. C.

Ukhorskiy, A. Y., B. J. Anderson, P. C. Brandt, and N. A. Tsyganenko (2006), Storm time evolution of the outer radiation belt: Transport and losses, J. Geophys. Res., 111, A11S03, doi:10.1029/2006JA011690.

Varotsou, A., D. Boscher, S. Bourdarie, R. B. Horne, S. A. Glauert, and N. P. Meredith (2005), Simulation of the outer radiation belt electrons near geosynchronous orbit including both radial diffusion and resonant interaction with whistler mode chorus waves, Geophys. Res. Lett., 32, L19106, doi:10.1029/2005GL023282.

Xiao, F., Z. Su, H. Zheng, and S. Wang (2009a), Modeling of outer radiation belt electrons by multidimensional diffusion process, J. Geophys. Res., 114, A03201, doi:10.1029/2008JA013580.

Xiao, F., Q.-G. Zong, and L. Chen (2009b), Pitch-angle distribution evolution of energetic electrons in the inner radiation belt and slot region during the 2003 Halloween storm, J. Geophys. Res., 114, A01215, doi:10.1029/2008JA013068.

Xiao, F. Z. S., H. Zheng, and S. Wang (2010), Three-dimensional simulations of outer radiation belt electron dynamics including cross-diffusion terms, J. Geophys. Res., 115, A05216, doi:10.1029/2009JA014541.

V. Angelopoulos, Institute for Geophysics and Planetary Physics, University of California, Box 951567, Los Angeles, CA 90095, USA

J. W. Bonnell, Space Sciences Laboratory, University of California, 7 Gauss Way, Berkeley, CA 94720-7450, USA

S. R. Elkington, Laboratory for Atmospheric and Space Physics, University of Colorado, 1234 Innovation Dr., Boulder, CO 80303, USA.

I. R. Mann, Department of Physics, University of Alberta, MS 615 , Edmonton, AB T6G 2G7, Canada.

T. M. Loto'aniu and H. J. Singer, Space Weather Prediction Center, National Oceanic and Atmospheric Administration, 325 Broadway, Boulder, CO 80305, USA. (paul.lotoaniu@noaa.gov)

C. L. Waters, Center for Space Physics, School of Mathematical and Physical Sciences, University of Newcastle, University Dr., Newcastle, NSW 2308, Australia. 